\title{
Effectiveness of muscle vibration in modulating corticospinal excitability
}

\author{
Lorraine Smith, MSc; Brenda Brouwer, PhD* \\ Motor Performance Laboratory, School of Rehabilitation Therapy, Queen's University, Kingston, Ontario, Canada
}

\begin{abstract}
This study explored the effect of vibration of the forearm extensors on motor cortical excitability and the influence of stimulus duration. Sixteen healthy volunteers between 23 and 42 years old participated in one or two studies. We applied 15 or 30 min of $100 \mathrm{~Hz}, 0.5 \mathrm{~mm}$-amplitude vibration to the extensor carpi radialis longus (ECRL) muscle. Cortical excitability was measured as the magnitude of the motorevoked potentials (MEPs), and the size of the representation area associated with ECRL and flexor carpi ulnaris muscles was determined with the use of transcranial magnetic stimulation. A $33 \%$ increase in MEP size and enlarged area of cortical excitability was detected 5 min after 15 min of vibration in the ECRL only. No changes were associated with $30 \mathrm{~min}$ of vibration in either muscle. These findings indicate that the facilitatory effects of vibration in healthy subjects depend on stimulus duration and provide impetus for testing the extent to which shortduration vibration augments corticospinal excitability to improve muscle function in people with central motor disorders.
\end{abstract}

Key words: facilitation, motor cortex, motor-evoked potential, plasticity, proprioception, rehabilitation, reorganization, spindle afferents, transcranial magnetic stimulation, upper limb.

\section{INTRODUCTION}

In neurorehabilitation, the application of proprioceptive, cutaneous, electrical, and vibratory stimulation is used to promote normal patterns of motor activity by modulation of the excitability of motoneurons projecting to target muscles [1-3]. The criteria for making decisions about which technique may produce the best results, however, remain elusive, partly because the underlying physiological mechanisms are not well understood [3].

Motor cortical organization can be remodeled secondary to altering afferent input, as evidenced by changes in the size of the descending volley originating from the motor cortex and modulation of the cortical representation area associated with the region stimulated [2,4-5]. Electrical stimulation of the ulnar nerve at motor threshold resulted in marked increases in the amplitude of the motor-evoked potentials (MEPs) recorded from ulnar innervated muscles only in response to transcranial magnetic stimulation (TMS) [4]. The absence of change in the persistence or amplitude of F-waves generated from the same muscles suggested that reorganization occurred at

\footnotetext{
Abbreviations: ANOVA $=$ analysis of variance, $\mathrm{C} 4=$ central position along the coronal line on scalp, optimal stimulation site; ECRL = extensor carpi radialis longus, EMG = electromyographic, FCU = flexor carpi ulnaris, ICC $=$ intraclass correlation coefficient, LICI = long-interval intracortical circuit, MEP = motor-evoked potential, SD = standard deviation, SICI = shortinterval intracortical inhibition, TES = transcranial electrical stimulation, TMS = transcranial magnetic stimulation, vib15 = 15 min of vibration, vib30 $=30$ min of vibration.

This material was the result of work supported with resources provided partly by the Botterell Foundation, Queen's University, Kingston, Ontario, Canada.

*Address all correspondence to Brenda Brouwer, PhD, Professor; School of Rehabilitation Therapy, Queen's University, 31 George Street, Kingston, Ontario, Canada K7L 3N6; 613-5336087; fax: 613-533-6776. Email: brouwerb@post.queensu.ca DOI: 10.1682/JRRD.2005.02.0041
} 
the cortical level. Further, since reorganizational changes were not evident in response to subthreshold ulnar nerve stimulation or cutaneous stimulation of digital nerves, researchers concluded that muscle afferents are important in promoting targeted cortical plasticity $[4,6]$.

Vibration is perhaps the most potent mechanical stimulus for activating primary spindle afferents [7-8]. Kossev et al. [9] reported an almost twofold increase in MEP amplitude in response to TMS applied immediately after briefduration, low-amplitude vibration of the extensor carpi radialis longus (ECRL) muscle at a frequency of $80 \mathrm{~Hz}$. Others have confirmed that the excitability of corticospinal projections to the target muscle increases during vibration but also have indicated that the excitability to other nearby muscles is suppressed [10-11]. Evidence has shown that vibration frequencies up to $120 \mathrm{~Hz}$ are effective at augmenting MEP amplitude [11], although little is known about the importance of other stimulus parameters. For example, Fraser et al. [2] demonstrated that cortically evoked activation of pharyngeal muscles is optimally enhanced following 10 min of electrical stimulation compared with shorter and longer periods. With respect to muscle vibration, the extent to which changes in cortical excitability are influenced by the period of application is not known. It is, however, well established that long periods of repeated sensory input result in significant reorganization of sensorimotor representations in experimental animals and humans [4,12-13].

Clinically, it is important to understand the stimulus characteristics that promote optimal results and, in the case of corticospinal excitability, to determine whether changes are sustained beyond the stimulation period to enable exploitation. As an initial step, this observational cohort study examined the effectiveness of vibration applied to the ECRL muscle for a total of 15 or $30 \mathrm{~min}$ in promoting changes in the excitability of corticospinal projections in healthy subjects. The findings will help form the basis for establishing a protocol that can be applied and tested in a clinical setting involving patients with central motor disorders.

\section{MATERIALS AND METHODS}

\section{Subjects}

Sixteen healthy volunteers were recruited by word of mouth and in response to posted advertisements. They ranged in age from 23 to 42 years and participated in one or more experiments. The sample size required was determined based on a significance level of 0.05 and a power of 0.80 to detect changes in cortical excitability of 20 percent from baseline [4]. Variance was assumed to be 15 percent, which is consistent with the reproducibility of MEP amplitudes obtained from relaxed subjects in response to TMS [14]. Ten subjects were required for each protocol.

We screened subjects to ensure that they had no selfreported injuries or pain affecting the upper limbs and that they had no contraindication for TMS (e.g., cardiac pacemaker, intracranial implants, history of epilepsy [15]). All participants provided informed consent, and all procedures were approved by the Queen's University Human Research Ethics Board.

\section{Measuring Cortical Excitability}

All subjects underwent TMS mapping of the left motor cortex with the use of a Cadwell MES-10 electromagnetic stimulator (Cadwell Laboratories, Kennewick, Washington). Subjects were positioned semireclined on a padded plinth and were asked to wear a bathing cap pulled taut over their scalp. The optimal scalp site for eliciting MEPs in the ECRL muscle with the use of a figure-eight coil (8 cm external wing diameter) was determined with an initial stimulus intensity of 60 percent. The coil first positioned $3 \mathrm{~cm}$ lateral to the vertex and three stimuli delivered. We then moved the coil in a $1 \mathrm{~cm}$ grid pattern to identify the location at which the largest stable MEPs were observed; this optimal position was marked on the cap. To determine motor threshold, we decreased the stimulus intensity in steps of 5 percent as long as MEPs were observed. When MEPs were no longer elicited, we increased the intensity 3 percent, then adjusted it upward or downward in steps of 1 percent as appropriate to establish the lowest stimulus intensity required to evoke 5 MEPs with minimum amplitudes of $50 \mathrm{~V}$ in response to 10 stimuli [16].

Following the protocol described by Ridding et al. for mapping cortical excitability [4], we marked six additional stimulation sites at $1 \mathrm{~cm}$ intervals, three medial to the optimal stimulation site and three lateral to it. Each scalp site was stimulated in turn with five stimuli delivered at 3 to $5 \mathrm{~s}$ intervals at an intensity 20 percent above motor threshold. Corresponding MEPs elicited in the contralateral relaxed ECRL, and flexor carpi ulnaris (FCU) muscles were recorded by disposable silver-silver chloride electrodes placed in a bipolar configuration and referenced to a common ground electrode (a lip clip). The 
electromyographic (EMG) recordings were filtered (20 Hz to $2 \mathrm{kHz}$ ), amplified $(1,000 \times)$ and digitized ( $5 \mathrm{kHz}$ per channel) for a $70 \mathrm{~ms}$ period that included a 20 ms prestimulus portion. The five recordings from each stimulus site were averaged online (DataWave Technologies, Longmont, Colorado).

We monitored EMG activity on an oscilloscope throughout the experiment to ensure that muscles remained relaxed. In this way we were also able to ensure that the vibration did not elicit the tonic vibration reflex [17-18].

We conducted a second baseline mapping trial approximately 15 min later to assess the stability of the recordings, after which the vibration intervention was introduced. Mapping was repeated 5 min after the intervention and again 20 min later.

\section{Muscle Vibration}

The right forearm rested prone on an adjustable height table such that the arm was positioned in $20^{\circ}$ of shoulder abduction and $80^{\circ}$ of elbow flexion. We applied low-amplitude ( $0.5 \mathrm{~mm}$ peak-to-peak), high frequency $(100 \mathrm{~Hz})$ vibration to the belly of the ECRL, muscle in a $30 \mathrm{~s}$ on/15 s off duty cycle using a handheld muscle vibrator with a $1 \mathrm{~cm}$ diameter contact surface (Wahl Corporation, Model 4180, 11W, Sterling, Illinois) that is commonly used in clinical settings [19]. The stimulus parameters were compatible with other protocols reported in the literature and enabled one-to-one discharges of Group Ia spindle afferents [7-8,11] while avoiding the tonic vibration reflex $[17,20]$. Vibration was applied for either $15 \mathrm{~min}$ or $30 \mathrm{~min}$.

\section{Data Analysis}

We rectified the average MEPs evoked from each muscle in response to stimulation at each scalp site and calculated the areas to provide a measure of corticospinal excitability. This measure is referred to as MEP size. The consistency of the MEPs recorded over time prior to introducing vibration was determined from the intraclass correlation coefficient $\left(\operatorname{ICC}_{(3, k)}\right)$, which reflects the agreement and correspondence between measurements. An ICC of 0.75 was considered acceptable for outcomes used to demonstrate change [21].

Since only four subjects received both $15 \mathrm{~min}$ (vib15) and 30 min of vibration (vib30), it was important to establish the two groups at baseline. We used $t$-tests to compare motor thresholds and MEP size between vib15 and vib30 groups. To address the questions of whether vibration induced changes in corticospinal excitability and if the duration of application was important, we used a mixed analysis of variance (ANOVA) including withinsubject factors of time (before and two time points after vibration), muscle (ECRL and FCU), and stimulus site (7), and one between-subjects factor of group (vib15 and vib30). We adopted a $p$-value of 0.05 to test the significance of main effects of each factor and all interactions between factors. If necessary, we performed post hoc analyses (Tukey) to establish where differences lay.

We analyzed the scalp sites from which MEPs were elicited in the wrist extensors to identify changes in cortical representation area. A two-factor ANOVA with time as a repeated measure and group as the between-subject factor was used.

All statistical analyses were performed with the Statistical Package for the Social Sciences (SPSS, Chicago, Illinois). In all cases, descriptive statistics are reported, in mean \pm 1 standard deviation (SD) unless otherwise indicated.

\section{RESULTS}

All participants $(n=16)$ completed one or both vibration protocols (i.e., vib15 or vib30). Six men and four women (mean age $=26.7 \pm 3.2$ years) constituted the vib15 group and four men and six women (mean age = $28.6 \pm 6.2$ years) made up the vib30 group; four individuals took part in both (3 men and 1 woman), 1 to 2 weeks apart. The mean motor threshold was similar for both groups (vib15: $46.5 \pm 7.5 \%$, vib30: $42.6 \pm 8.0 \%$; $p=$ 0.350 ). Indeed, the motor thresholds of those subjects involved in both protocols differed by less than 2 percent. The baseline MEPs from ECRL and FCU were also similar in size for both groups ( $p \geq 0.232)$.

\section{Consistency of MEPs}

The reliability of MEP size measured prior to vibration was very good, as reflected by an ICC of 0.77 . We subsequently used an average of the baseline MEP measures in all analyses.

\section{Muscle Vibration}

Analysis of main between-subject effects revealed, as expected, a significant effect of stimulus site $\left(F_{6,252}=7.85\right.$, $p<0.001$ ), showing that MEPs were largest when evoked from the optimal scalp site (C4 [optimal stimulation site]) and progressively diminished as TMS was applied more medially or laterally. Post hoc analysis confirmed that TMS 
applied at C4 produced larger MEPs than those associated with stimulation sites $\geq 2 \mathrm{~cm}$ away $(p \leq 0.007)$. Also not surprising was that MEPs were larger in the target muscle ECRL than in $\operatorname{FCU}\left(F_{1,252}=73.44, p<0.001\right)$.

Of particular relevance was the significant main effect of group $\left(F_{1,252}=6.52, p=0.011\right)$. The larger MEPs evident in the vib15 group reflected the augmentation of MEPs after vibration in this group only (withinsubject effects); no changes were found in MEPs in the vib30 group (time $\times$ group interaction $F_{2,504}=6.21, p=$ 0.002). The failure to detect a main effect of time $\left(F_{2,504}=2.18, p=0.113\right)$ paired with a significant interaction between time, group, and muscle $\left(F_{2,504}=5.49\right.$, $p=0.004$ ) further demonstrates the differential effects associated with the duration of vibration. The selective increase in ECRL activation by about 33 percent following $15 \mathrm{~min}$ of vibration (Figure $\mathbf{1}$ is a representative example), however, was not sustained 20 min later as MEPs returned to baseline values. A summary of the results is presented in Figure 2.

In addition to the findings just mentioned was an expansion of the cortical representation area associated with the ECRL, which increased from $4.3 \pm 0.9$ scalp sites producing MEP responses at baseline to $5.4 \pm 1.2$ postvibration $(p<0.020)$ in the vib15 group. The increased region of cortical excitability was not sustained 20 min postvibration. No changes were detected in cortical representation of the ECRL in the vib30 group (baseline: $4.0 \pm 1.0$; postvibration: $4.2 \pm 1.6$; $p=0.509$ ) and the representation area associated with FCU remained constant for both groups throughout the test period (vib15: $p=0.333$; vib30: $p=0.472$ ).

In light of the differences between the vib15 and vib30 groups, we wanted to ensure that vibration was exerting the expected effect during application (i.e., increased motoneuronal excitability). Half the subjects in the vib30 group agreed to return 1 week later, during which time MEPs were recorded from the ECRL muscles in response to TMS applied over the optimal scalp site only. Recordings obtained at baseline and at 5 min intervals throughout the application of vibration indicated that in all cases $(n=5)$, MEPs were augmented during vibration, with the exception of the final observation. At $30 \mathrm{~min}$, the MEPs of three subjects decreased below baseline values, and in the remaining subjects, the MEPs were smaller than those evident at any other time during vibration (Figure 3).
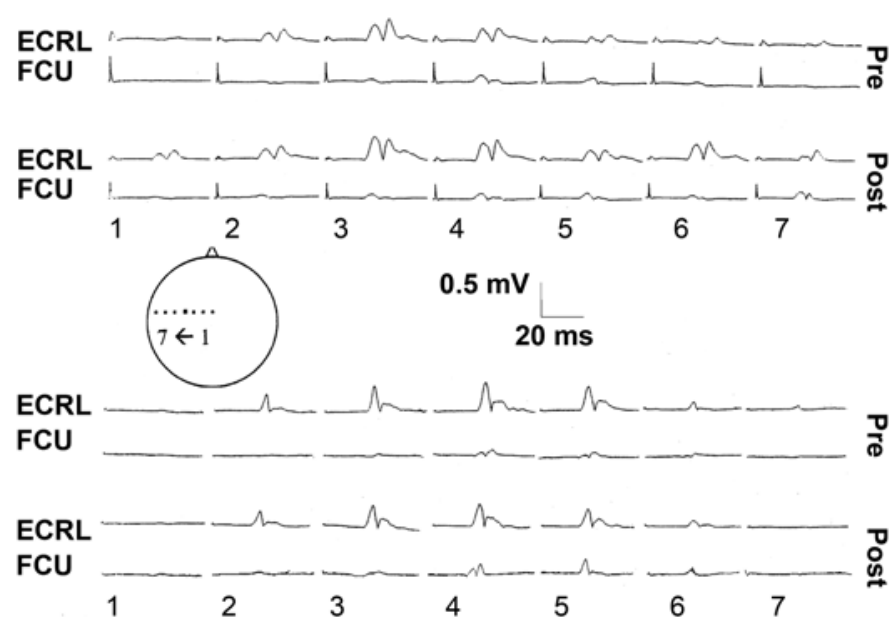

Figure 1.

Representative examples of motor-evoked potentials recorded preand postvibration applied for $15 \mathrm{~min}$ (top) and $30 \mathrm{~min}$ (bottom). All traces are from same subject; protocols were introduced 1 week apart. ECRL = extensor carpi radialis longus; FCU = flexor carpi radialis.

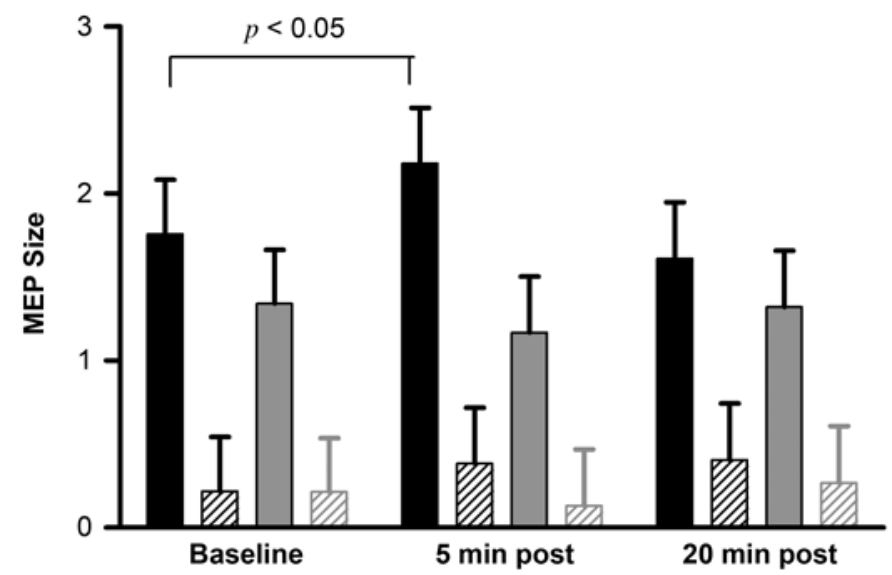

Figure 2.

Mean motor-evoked potential (MEP) size ( +1 standard error of mean) elicited in the extensor carpi radialis (solid) and flexor carpi ulnaris (hatched) muscles at baseline, $5 \mathrm{~min}$, and $20 \mathrm{~min}$ postvibration applied for 15 (black) and 30 (gray) min. Ten subjects per group.

\section{DISCUSSION}

The major finding of this study was that 15 min of vibration applied over the extensor surface of the forearm induced significant changes in motor cortical output shortly after the intervention. The effect was specific to the target muscle, as evidenced by a 33 percent increase in MEP size and an enlarged region of cortical excitability 

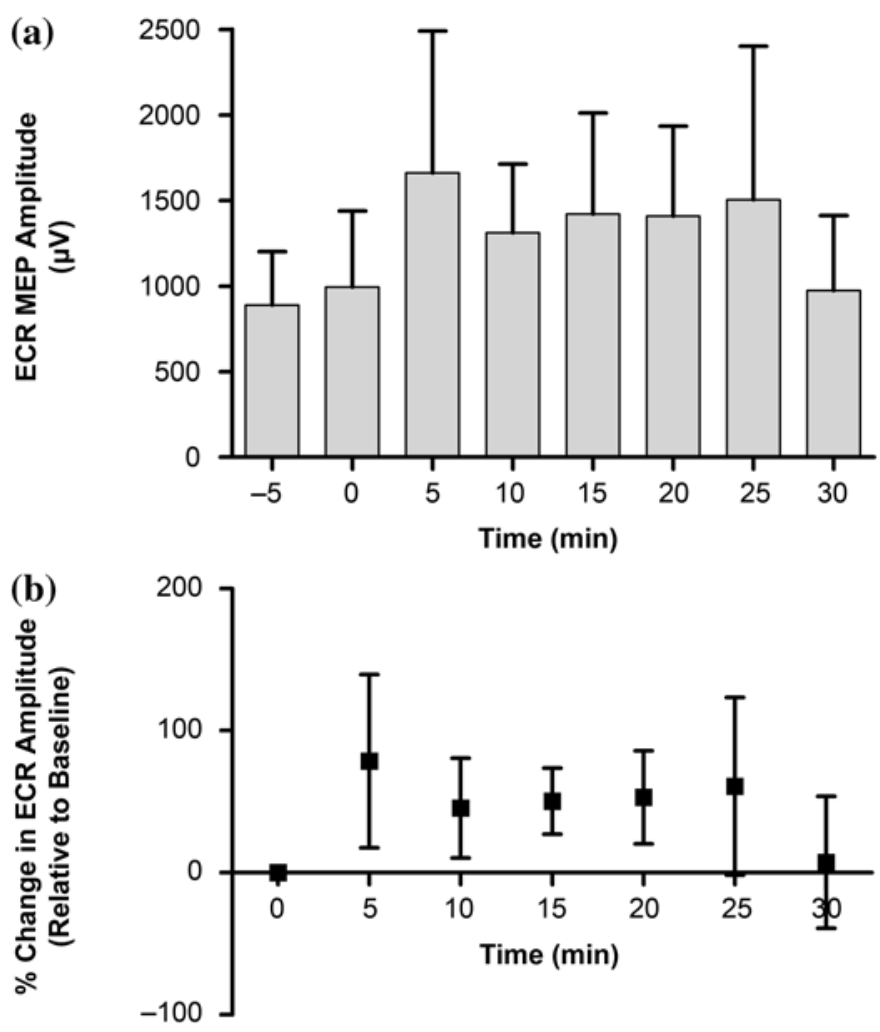

Figure 3.

(a) Mean (+1 standard deviation) extensor carpi radialis (ECR) motor-evoked potential (MEP) amplitude at baseline and at 5 min intervals during $30 \mathrm{~min}$ of vibration. (b) MEPs expressed as a percentage of baseline; error bars are \pm 1 standard deviation. Five subjects total.

associated with the ECRL muscle only. Prolonging the application of vibration failed to produce greater MEP facilitation and may have even decreased excitability, although not significantly so.

Literature has well established that periods of augmented sensory input introduced with vibration markedly increase the excitability of neural circuits that control the motor output to the stimulated muscle [9-11,18,22-24]. The increased MEP size found in the current study is consistent with such reports and in addition demonstrated expansion of the cortical representation area associated with the vibrated ECRL muscle. Furthermore, we found that vibration-induced cortical modulation was sustained up to 5 min beyond the 15 min stimulation period. Arguably, we did not examine whether the changes could have been due to alterations in spinal excitability alone, although many others have provided convincing evi- dence that vibration applied as in the current study results in modulations at the cortical level [9-10,18,20]. For example, Kossev and colleagues showed that MEP facilitation induced by muscle vibration was evident after TMS but not transcranial electrical stimulation (TES) [9]. Since TES activates corticomotoneurons at the axon hillock, whereas TMS activates cortical cells transynaptically [25-26], the vibration-induced augmentation of MEPs following TMS only indicates a cortical origin [9].

Driving changes in motor cortical output through specific inputs has tremendous implications for rehabilitation if associated with improved function. Demonstration of such effects in clinical populations, however, is extremely limited, in part because little is known about the optimal stimulus parameters required to promote cortical plasticity [2]. Also, the inherent heterogeneity among patient populations with neural impairments can preclude the identification of consistent or robust TMS response patterns [27]. In healthy subjects, we determined that prolonging the duration of vibration offered no benefit and, in fact, healthy subjects suffered a detriment in their ability to enhance corticospinal excitability. It should be noted that subjects in both groups were recruited from the university population and were similar in age, handedness, and baseline measures; thus, the differential effects of 15 and 30 min of vibration most likely cannot be explained on the basis of selection bias.

Beyond 15 min of vibration, the degree to which MEPs were facilitated was highly variable (Figure 3), but only after 25 min was the effect reversed in some subjects. While the mechanisms underlying these effects were not addressed, others have suggested that changes in levels of intracortical inhibition can alter the effect of afferent stimulation $[2,10,18,24]$. The GABAergic inhibitory circuits are important for the maintenance and adjustment of motor cortical representations [28]. Rosenkrantz and Rothwell demonstrated reductions in shortinterval intracortical inhibition (SICI) following vibration of the intrinsic hand muscles and, furthermore, that these circuits are altered by long-interval intracortical circuits (LICIs) [10,18]. We speculate that after 15 min of vibration, the SICI was down-regulated, resulting in enlargement of the ECRL representation area, but with prolonged vibration the LICI may have effectively cancelled the SICI effect [10].

Similar reasoning was used to explain the attenuation of responsiveness of cortical neurons as they adapted to the stimulus characteristics following exposure to prolonged, 
repetitive stimulation [29]. GABAergic pathways were implicated in the gradual depression of synaptic transmission, thus reducing the response to stimulation; the excitability at the cellular level remained unchanged. The reversal of the vibration effect on cortical excitability observed in the present study may reflect a comparable mechanism altering the balance of excitatory and inhibitory influences. Afferent feedback is known to modulate intracortical inhibition; therefore, it follows that if the responsiveness of spindle afferents is diminished following prolonged vibration (30 min) that this might be expressed as a relative reduction in cortical excitability [11,24]. Further study is required to identify the mechanisms involved.

It should be noted that the magnitude of change in cortical excitability detected following short-term vibration was less than that reported by others. The most logical explanation is that the effects diminish with time as indicated by the return to prestimulus levels within 20 min. Previous studies have found increases in MEP amplitude ranging between 34 and 194 percent within seconds of vibration cessation [9-11,18,24]. Figure 3(b) shows comparable mean increases of 45 to 80 percent were evident during the vibration period, and we extend these observations by demonstrating that cortical excitability remains significantly enhanced several minutes beyond stimulus cessation. This increased excitability of corticospinal projections can be important clinically, particularly for patients severely limited by paresis if accompanied by improved muscle function. Further study involving patients is required to determine if these short-term adaptations are reproducible and can be exploited to promote more normal muscle activation not only during vibration but also for a period afterward.

\section{CONCLUSION}

We conclude that the excitability of motor cortical projections can be augmented in healthy subjects in response to muscle vibration, but the effect depends on the duration of stimulation. Vibration for $15 \mathrm{~min}$ increases both the size of the corticospinal volley and the cortical representation area of the target muscle, which is maintained for at least $5 \mathrm{~min}$. We did not address whether modification of other stimulus parameters could promote greater cortical reorganization, since most clinically available vibrators have fixed frequencies and amplitudes consistent with the present study [19]. The application of vibration for neuromuscular rehabilitation warrants exploration in view of the current findings.

\section{REFERENCES}

1. Bishop B. Vibration stimulation I. Neurophysiology of motor responses evoked by vibratory stimulation. Phys Ther. 1974;54:1273-82.

2. Fraser C, Power M, Hamdy S, Rothwell J, Hobday D, Hollander I, Tyrell P, Hobson A, Williams S, Thompson D. Driving plasticity in human adult motor cortex is associated with improved function after brain injury. Neuron. 2002;34(5):831-40.

3. Hummelsheim H, Hauptmann S, Neumann S. Influence of physiotherapeutic facilitation techniques on motor evoked potentials in centrally paretic hand extensor muscles. Electroencephalogr Clin Neurophysiol. 1995;97(1):18-28.

4. Ridding MC, Brouwer B, Miles TS, Pitcher JB, Thompson $\mathrm{PD}$. Changes in muscle responses to stimulation of the motor cortex induced by peripheral nerve stimulation in human subjects. Exp Brain Res. 2000;131(1):135-43.

5. Ridding MC, Rothwell JC. Afferent input and cortical organisation: A study with magnetic stimulation. Exp Brain Res. 1999;126(4):536-44.

6. Dobkin BH. Do electrically stimulated sensory inputs and movements lead to long-term plasticity and rehabilitation gains? Curr Opin Neurol. 2003;16(6):685-91.

7. Burke D, Hagbarth KE, Lofstedt L, Wallin BG. The responses of human muscle endings to vibration of noncontracting muscles. J Physiol. 1976;261(3):673-93.

8. Roll JP, Vedel JP, Ribot E. Alteration of muscular proprioceptive messages by induced tendon vibration in man: A microneurographic study. Exp Brain Res. 1989;76(1):213-22.

9. Kossev A, Siggelkow S, Schubert M, Wohlfarth K, Dengler R. Muscle vibration: Different effects on transcranial magnetic stimulation and electrical stimulation. Muscle Nerve. 1999;22(7):946-48.

10. Rosenkranz K, Rothwell JC. Differential effect of muscle vibration on intracortical inhibitory circuits in humans. J Physiol. 2003;551(Pt 2):649-60.

11. Siggelkow S, Kossev A, Schubert M, Kappels HH, Wolf W, Dengler R. Modulation of motor evoked potentials by muscle vibration: The role of vibration frequency. Muscle Nerve. 1999;22(11):1544-48.

12. Menning H, Roberts LE, Pantev C. Plastic changes in the auditory cortex induced by intensive frequency discrimination training. Neuroreport. 2000;11(4):817-22.

13. Recanzone GH, Jenkins WM, Hradek GT, Merzenich MM. Progressive improvement in discriminative abilities in adult owl monkeys performing a tactile frequency discrimination task. J Neurophysiol. 1992;67(5):1015-30. 
14. Mortifee P, Stewart H, Schulzer M, Eisen A. Reliability of transcranial magnetic stimulation for mapping the human motor cortex. Electroencephalogr Clin Neurophysiol. 1994; 93(2):131-37.

15. DeLisa JA, Lee HJ, Baran EM, Lai KS. Manual of nerve conduction velocity and clinical neurophysiology. 3rd ed. New York (NY): Lippincott, Williams, \& Wilkins; 1994.

16. Brouwer B, Hopkins-Rosseel DH. Motor cortical mapping of proximal upper extremity muscles following spinal cord injury. Spinal Cord. 1997;35(4):205-12.

17. Marsden CD, Meadows JC, Hodgson HJ. Observations on the reflex response to muscle vibration in man and its voluntary control. Brain. 1969;92(4):829-46.

18. Rosenkranz K, Rothwell JC. The effect of sensory input and attention on the sensorimotor organization of the hand area of the human motor cortex. J Physiol. 2004;561(Pt 1):307-20.

19. Turnbull GI, Ross LC, Peacock JB. Frequency analysis of commercially available vibrators. Physiother Can. 1982; 34(1):21-26.

20. Rollnik JD, Siggelkow S, Schubert M, Schneider U, Dengler R. Muscle vibration and prefrontal repetitive transcranial magnetic stimulation. Muscle Nerve. 2001;24(1):112-15.

21. Portney LG, Watkins MP. Statistical measures of reliability. In: Portney LG, Watkins MP, editors. Foundations of clinical research: Applications to practice. 1st ed. Norwalk (CT): Appleton \& Lange; 1993. p. 505-28.

22. Claus D, Mills KR, Murray NM. The influence of vibration on the excitability of alpha motoneurons. Electroencephalogr Clin Neurophysiol. 1988;69(5):431-36.
23. Claus D, Mills KR, Murray NM. Facilitation of muscle responses to magnetic brain stimulation by mechanical stimuli in man. Exp Brain Res. 1988;71(2):273-78.

24. Rosenkranz K, Pesenti A, Paulus W, Tergau F. Focal reduction of intracortical inhibition in the motor cortex by selective proprioceptive stimulation. Exp Brain Res. 2003; 149(1):9-16.

25. Day BL, Dressler D, Maertens de Noordthout A, Marsden CD, Nakashima K, Rothwell JC, Thompson PD. Electrical and magnetic stimulation of the human motor cortex: Surface EMG and single motor unit responses. J Physiol. 1989;412:449-73. Erratum in: J Physiol (Lond) 1990;430: 617.

26. Edgley SA, Eyre JA, Lemon RN, Miller S. Excitation of the corticospinal tract by electromagnetic and electrical stimulation of the scalp in the macaque monkey. J Physiol. 1990;425:301-20.

27. Hendricks HT, Zwarts MJ, Plat EF, van Limbeek J. Systematic review for the early prediction of motor and functional outcome after stroke by using motor-evoked potentials. Arch Phys Med Rehabil. 2002;83(9):1303-8.

28. Jacobs KM, Donoghue JP. Reshaping the cortical motor map by unmasking latent intracortical connections. Science. 1991;251(4996):944-47.

29. Eytan D, Brenner N, Marom S. Selective adaptation in networks of cortical neurons. J Neurosci. 2003;23(28):9349-56.

Submitted for publication February 10, 2005. Accepted in revised form August 9, 2005. 